\title{
Absence management and presenteeism: the pressures on employees to attend work and the impact of attendance on performance
}

Denise Baker-McClearn, Kay Greasley, Jeremy Dale and Frances Griffith, Health

Sciences Research Institute, Warwick Medical School, University of Warwick

Human Resource Management Journal, Vol 20, no 3, 2010, pages 311-328

Absenteeism is an issue that has grown in importance over the past few years; however, little has been done to explore the impact of presenteeism on individual and organisational performance and well-being. This article is based on interviews collected in nine case study organisations in the UK. Two sector organisations (one private and one public) were studied to examine absence management and a conceptual model of presenteeism, with further illustration provided using data from the other seven case studies. This enabled a pattern of presenteeism to emerge, along with the contextual and individual factors which impact on it. In addition to previous research, we found that presenteeism is a complex 'problem' and that it is not a single one-dimensional construct, but is continually being shaped by individual and organisational factors. In addition, we found that performance and well-being are more closely related to the organisational reaction to presenteeism and absenteeism, rather than the act itself. Contact: Dr Denise Baker-McClearn, Health Sciences Research Institute, Warwick Medical School, University of Warwick, Coventry CV4 7AL, UK. Email: denise.baker@warwick.ac.uk

\section{INTRODUCTION}

$\mathbf{0}$ ptimising workforce health, the prevention of work- and lifestyle-related illness and managing absenteeism are issues that have grown significantly in importance over the past few years and are identified as public health and economic priorities. In the UK, Black's (2008) recent review of the health of Britain's working age population, 'Working for a healthier tomorrow', suggests that the development of a wellness, rather than a sickness, culture is essential to reduce absence and improve performance in the workplace. She suggests that such a culture requires good line management, support within the workplace and a change in the perception that work is damaging except for those who are totally fit. Waddell and Burton (2006) have similarly argued that the 'right' kind of work can be good for a person, but what is less frequently described is what the 'right' kind of working environment entails and how factors in the work environment, including policies, culture and perceptions of absence management impact on employee absence and presence (including presenteeism) at work.

As early as 1978, Steers and Rhodes voiced concerns about the impact of attending work when sick, suggesting that some absenteeism can be good for an organisation while overreliance on absence figures as a measure of productivity can be counter-productive with unfavourable consequences for organisations and employees alike (Steers and Rhodes, 1978). The term presenteeism has been in usage for many years, although its definition is rather vague. For example, the trade union Unison (1999) used it to describe people 'who, despite complaints and ill health that should prompt rest and absence from work, are still turning up 
at their jobs' (Aronsson et al., 2000: 503). Some studies define presenteeism as a reduction in productivity because of health-related conditions (Schultz and Edington, 2007); this is a different focus, not considered here.

This article briefly outlines current understanding of absence from the workplace as this forms part of the context of presenteeism. It then reviews the existing literature on presenteeism, drawing out the key themes into a model clarifying the multifaceted nature of this issue. This model is used as the framework for analysis of interview data collected during the course of a study on well-being at work. The nuances in the data in relation to the way presenteeism plays out across a number of diverse organisations suggest that, although presenteeism may be influenced by organisational policy, individual thresholds for presenteeism fluctuate. We step back from studies measuring the extent of the phenomenon (e.g. Caverley et al., 2007) to look at its characteristics and to suggest how it reflects organisational and individual antecedents.

\section{ABSENCE FROM THE WORKPLACE}

Absenteeism has been described as the single largest source of lost productivity in business and industry in the UK, with minor illness causing the most short-term absence and stress, and mental health issues causing the most absence of over 4 weeks duration (CIPD Survey, 2005). The costs of absence are not only borne by organisations, in terms of health-related productivity losses resulting from staff absence, staff turnover, loss of skill base, recruitment and retraining, but also by the general population through costs to the health service (estimated at $£ 5-11$ billion a year), the government through supplying government benefits ( $£ 29$ billion a year) and the loss of additional income through taxes of those off sick ( $£ 28-36$ billion).

Monitoring absence and supporting health and well-being improves productivity and lowers absence levels, and may reduce the length of current and subsequent periods of absence (James et al., 2002). Several large organisations, such as Mondial (Jenneh, 2006), Toshiba (Pollitt, 2006) and Cadbury Trebor Bassett (Pollitt, 2007), have implemented proactive health and absence management policies intended to 'empower' employees to take responsibility for their own health and well-being. Research from Scandinavia supports this idea arguing that by being proactive, economic benefits are realised in the form of increased performance and productivity and a reduction in absence levels (Van Amelsvoort et al., 2006). How policies affect performance and employee well-being is not always evident. Grinyer and Singleton (2000) suggested that simply striving to reduce absence rates without focusing on those who attend work and under-perform produces artificially low absence figures and fails to improve organisational or individual efficiency. A balance, therefore, may be needed between managing absence and ensuring employees are able to perform adequately.

\section{PRESENTEEISM}

Presenteeism is increasingly being seen as a threat to employee efficiency and workplace safety. Although employers' groups have often been reluctant to address problems of presenteeism, individual employers are beginning to take the issue more seriously with the establishment of occupational health and flexible working. In this section, we review the international literature on presenteeism, drawing out the key themes to develop a model (see Figure 1) for use in further analysis.

A UK mixed method study by Grinyer and Singleton (2000) compared two public sector offices, one with higher long-term sickness than the other. Organisations were matched on key 
FIGURE 1 Influences on presenteeism: a summary from published studies

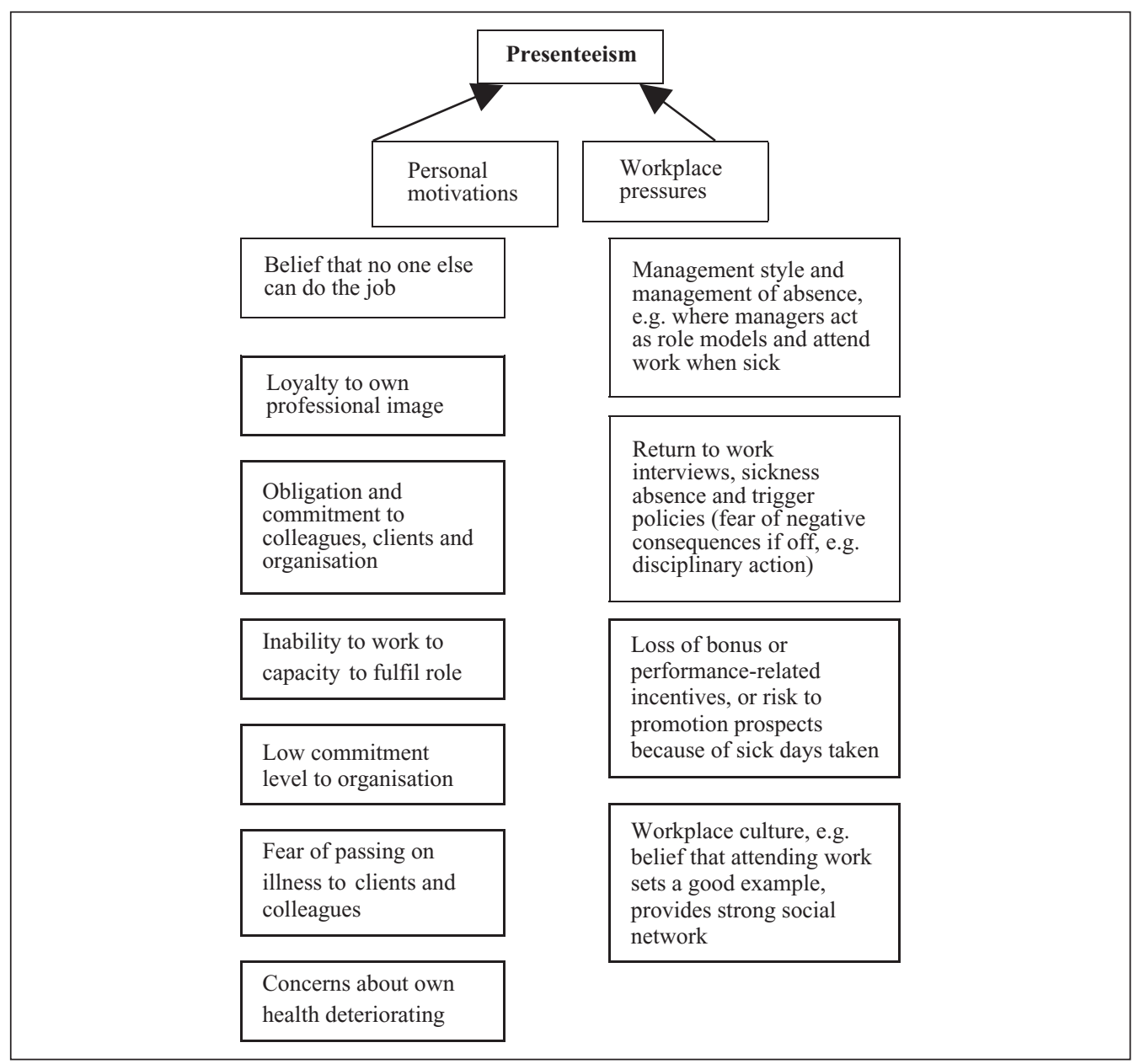

criteria such as size, employee profile and location, and employees were interviewed regarding two specific organisational factors related to the non-use of sick leave: obligation to colleagues (an internal pressure) and fear of reaching 'trigger' points (an external pressure). Work attendance was greater in those who felt strongly committed to serving others (clients or patients) or felt their absence would have negative consequences for themselves, colleagues or a third party. Organisational policies introduced to reduce 'casual sickness', such as increased monitoring of short-term sick leave, made staff fearful of being absent, which then left them feeling stressed and resentful. Policies were viewed as penalising the genuinely sick, as staff feared reaching a trigger point which would result in disciplinary action.

Chatterji and Tilley (2002), like Steers and Rhodes in the US, found in their mathematical modelling study that policies implemented to reduce absence, such as a reduction in sick pay, were more likely to increase presenteeism which in turn could lead to more illness and lower productivity. Taylor et al. (2003) concurred, suggesting that policies encouraging attendance at the cost of the employee adversely impact on employee morale and increase absence. Aronsson 
et al. (2000) and Hansson et al. (2006) in Scandinavia reported high presenteeism among organisations with a high 'attendance requirement' as employees felt they could not be replaced, often attending work when they were ill, in pain or before they were fully recovered.

In studies with public health-care workers in the US, New Zealand and Scandinavia, employees who worked directly with sick patients (Aronsson et al., 2000; Shamansky, 2002; Pilette, 2005) suffered a great deal of internal conflict over whether to attend work while suffering from what they saw as a contagious illness. Dixon (2005), in a US survey, reported that 56 per cent of employers felt 'presenteeism' was a problem in their organisation; employee burnout and lost productivity were 7.5 times greater with 'presenteeism' than absenteeism. Samuel and Wilson (2007) suggested that employers who encouraged their staff to take sick leave and were sympathetic to their illness promoted higher staff morale and loyalty as staff felt that the importance of their well-being was recognised.

Dew et al. (2005) compared two hospitals and a manufacturing site in New Zealand. For health-care workers, presenteeism was related to commitment to colleagues, loyalty to professional image and the institution, and the clients/patients within it. In the manufacturing organisation, attendance was strongly linked to management style and the economic pressures that drive business.

In the US, Ramsey (2006) found that work attendance was influenced by management style. Widespread 'presenteeism' occurred when managers and supervisors failed to take days off when sick themselves. Managers felt that they had to be brave and set a good example, and that they needed to be in work as their job could not be done by anyone else. The researchers, however, suggested that the adverse effect of this may be to spread contagious illnesses to other employees, therefore contributing to a greater loss of productivity and increased absence.

Concerns have been voiced about the impact of attending work with long-term health problems. Kivimaki et al. (2002) found from the Whitehall II survey in the UK that male civil servants who felt unhealthy and stressed and who failed to take sick leave were at greater risk of coronary events than those who took moderate amounts of sick leave, suggesting therefore that there is some evidence linking presenteeism to long-term health conditions. Caverley et al. (2007) found that presenteeism tended to be inversely related to absence levels; they argued that job reductions in their case study organisation had led to high levels of presenteeism.

In this study, we have examined the key influences within the workplace which appear to contribute to presenteeism and devised a model of these factors based on this literature (see Figure 1). These key influences can be divided into two groups: organisational pressures and personal motivations. We also identify in the model other mediating factors which impact on an individual's decision to attend work. We aim to understand how ideas of presenteeism are embedded in organisational practices. What does the concept mean to people? How does it connect to the management of attendance?

\section{THE STUDY}

This article reports the findings from the secondary analysis of interview data collected as part of a wider study of health and well-being in the workplace. The aim of this study was to evaluate the impact of proactive intervention(s) on attendance management, employee wellbeing and the organisational climate, focusing on the contextual issues which influence the successful introduction of proactive interventions. Interviews were conducted as part of an in-depth, multi-method case study of organisational approaches to employee health and attendance management. A detailed pre- and post-intervention evaluation, which examined the organisational profile prior to and following the implementation of a number of tailored, 
corporate level interventions, was conducted. Interventions were aimed at helping nine diverse organisations develop a more pro-active approach to improving the health and well-being of their workforce. The study then assessed the impact of health management programmes on organisational and employee performance.

Presenteeism emerged as a significant sub-theme during data analysis. In this article, we explore employees' accounts of presenteeism to understand the subtle differences between organisational intent as laid down in policy and procedure, and the individual interpretation of this intent by employees. As described below, the nine organisations all had distinct interests in promoting health and well-being, and they might be expected to follow good practice and to have little presenteeism when compared with the mass of organisations. In fact, the concept proved to be important in all of them.

\section{METHODS}

Semi-structured interviews were conducted with 123 people from nine organisations. In addition, policy documents were examined and during data collection, observational notes were made to supplement the interviews.

\section{Sampling}

A two-stage process was used to sample for 8-10 organisations to give maximum variation of organisations and within these, a range of employees which included blue collar and white collar, skilled and unskilled workers (Patton, 1990: 182).

Sampling organisations The project team approached 86 organisations with which they had professional contact or had worked with in the past. Fifty-four of these expressed initial interest but subsequently withdrew because of internal pressures, or restructuring that might impact on the implementation of the wider project, or failed to respond to subsequent communication.

Following detailed explanation about the study, 14 organisations committed to participate in the study. Information about each organisation was gathered in a structured telephone interview with the nominated lead. This was then used to select organisations to provide maximum variation across the secondary sampling criteria in Table 1 . The final sample consisted of two National Health Service (NHS) organisations, one police force, one local

TABLE 1 Criteria for organisation sampling

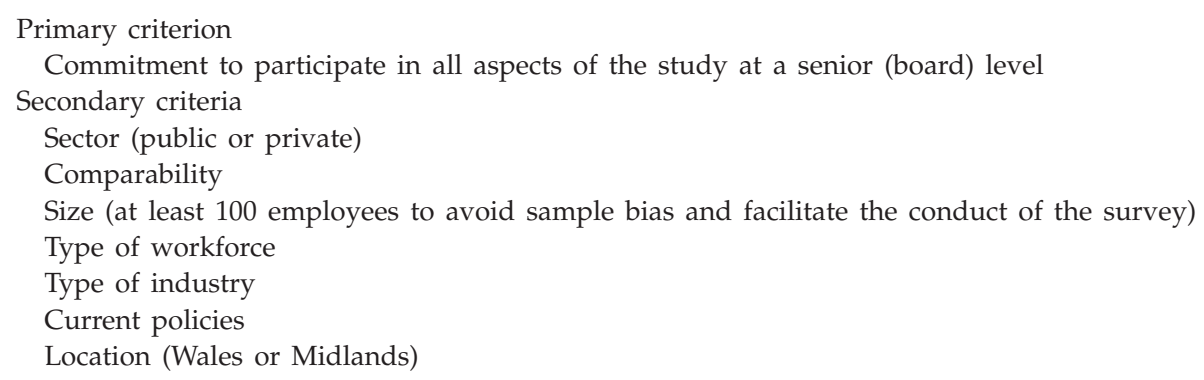


authority, two call centres, one manufacturing organisation, one power provider and one firm in heavy industry.

As the sample included an NHS organisation, we gained Multi-centre Research Ethics Committee approval and NHS Trust R\&D approval.

Sampling employees for interview For each organisation, a 'reference group' comprising representatives from Human Resources, Occupational Health, Management and Employee representatives was established to identify key stakeholders and individuals from within the organisation who might be willing to be interviewed. Interviewees were purposively sampled to reflect as diverse an array of personnel as possible. Each interviewee was informed of the purpose of the study, given time to consider whether they wished to participate and completed a consent form. Interviewee roles and organisations are listed in Table 2. A total of 123 interviews were conducted with 47 employees, 26 line managers/supervisors, 21 middle managers, 4 senior managers, 13 Human Resource employees, 9 Occupational Health employees and 3 Trade Union representatives.

\section{Interview process and data management}

Semi-structured interviews followed a topic guide (summarised in Table 3) which had been piloted in one organisation. Interviews were audio recorded and transcribed.

Transcriptions were checked for accuracy then imported into NVivo (QSR International, Stockport, UK) to assist with data handling. For the wider study, a coding frame was developed by DB and KG working on different sets of data within the study. Their coding frames and definitions were compared, combined and discussed with the research team. Codes were derived from the research questions and questions included in the topic guide or were secondary themes which emerged from the data. Constant refinement of the coding frame took place as the study progressed with stand-alone codes being added. For this article, the secondary theme of presenteeism was examined. Each author read at least five full transcripts where presenteeism appeared as an important theme. They then read the data which was coded under presenteeism. This article reports this data, but draws on the rest of the interview along with observation notes and organisational policy documents for understanding the context.

\section{Analysis process}

The policies of each organisation relevant to sickness absence were examined as the interview data needs to be interpreted for the context in which each interviewee was working. Two of the case study organisations, one private (CS1) and one public (CS2) sector organisation, were found to have high rates of reported presenteeism, as identified by interviewees who consistently reported this theme during interviews. We therefore initially focused on the rich data from these two organisations and compared it with the model in Figure 1. We then used the data from the other organisations to support or refute our analysis.

\section{FINDINGS}

\section{Policies relevant to sickness absence}

Here we provide a summary of the organisation's policies relevant to sickness absence. When reporting the interview data, we provide additional detail as needed.

We had no prior knowledge of each organisation's sickness absence policies, so diversity on this issue was not controlled for. We found that sickness absence policies had distinct 
TABLE 2 Interview sample by case study organisation

\begin{tabular}{|c|c|c|}
\hline Case study organisation & No. of interviewees & Interviewee details \\
\hline \multirow[t]{4}{*}{ CS1: Insurance: private } & 15 & 8 Employees \\
\hline & & 3 Supervisors \\
\hline & & 3 Managers \\
\hline & & 1 Human resources \\
\hline \multirow{5}{*}{ CS2: Health: public sector } & 19 & 9 Employees \\
\hline & & 3 Supervisors \\
\hline & & 3 Managers \\
\hline & & 1 Human resources \\
\hline & & 1 Occupational health \\
\hline \multirow{5}{*}{ CS3: Power: private } & 14 & 5 Employees \\
\hline & & 3 Team leaders \\
\hline & & 3 Managers \\
\hline & & 2 Human resources \\
\hline & & 1 Occupational health \\
\hline \multirow[t]{5}{*}{ CS4: Power: private } & 16 & 7 Employees \\
\hline & & 4 Team leaders \\
\hline & & 2 Managers \\
\hline & & 2 Human resources \\
\hline & & 1 Occupational health \\
\hline \multirow{6}{*}{ CS5: Call centre: private } & 11 & 4 Employees \\
\hline & & 1 Trade union employee \\
\hline & & 3 Supervisors \\
\hline & & 1 Manager \\
\hline & & 1 Occupational health \\
\hline & & 1 Human resources \\
\hline \multirow[t]{7}{*}{ CS6: Manufacturer: private } & 13 & 4 Employees \\
\hline & & 1 Trade union employee \\
\hline & & 3 Supervisors \\
\hline & & 1 Manager \\
\hline & & 1 Senior manager \\
\hline & & 2 Occupational health \\
\hline & & 1 Human resources \\
\hline \multirow[t]{7}{*}{ CS7: Local authority: public sector } & 14 & 4 Employees \\
\hline & & 1 Trade union employee \\
\hline & & 1 Occupational health employee \\
\hline & & 2 Supervisors \\
\hline & & 3 Managers \\
\hline & & 1 Senior manager \\
\hline & & 2 Human resources \\
\hline \multirow[t]{6}{*}{ CS8: Police force: public sector } & 12 & 4 Employees \\
\hline & & 2 Supervisors \\
\hline & & 2 Managers \\
\hline & & 2 Senior managers \\
\hline & & 1 Occupational health \\
\hline & & 1 Human resources \\
\hline \multirow[t]{5}{*}{ CS9: Health: public sector } & 11 & 2 Employees \\
\hline & & 3 Supervisors \\
\hline & & 3 Managers \\
\hline & & 2 Human resources \\
\hline & & 1 Occupational health \\
\hline
\end{tabular}


TABLE 3 Topic guide summary

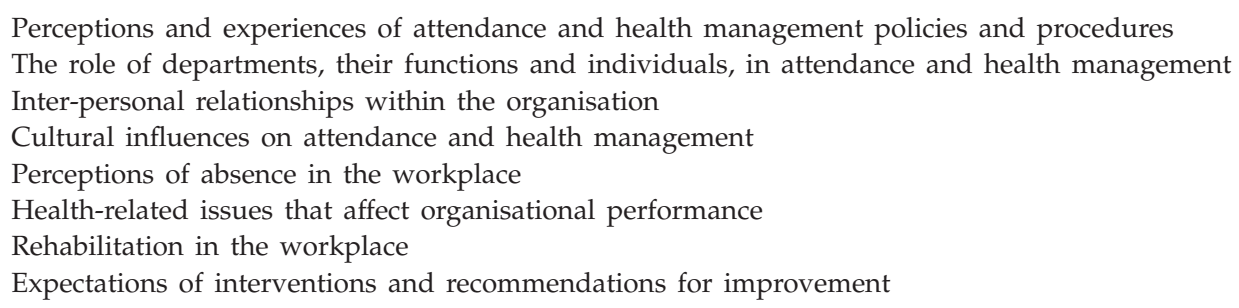

similarities; for example, they all had specific procedures for reporting absence, they all had stated periods of paid absence related to how long the employee had been working with the organisation; differences in the policies were generally related to how they were implemented and who was implementing them. All organisations had 'trigger' polices, where formal procedures were initiated if an employee had more than a certain number of absences (usually three) within a specific period of time (usually 12 months).

Sick pay entitlement was fairly standard for public sector organisations, but was more variable at private sector organisations. For example, at CS1, employees were only eligible for sick pay after 6 months of absence-free employment. They were also highly likely to have their sick pay suspended if they had more than three absences in a 12-month period. To have it reinstated, they had to have no absence for 6 months.

All organisations had procedures for rehabilitation which included phased return to work, or modified working, although employees often argued that some of the options were not offered to everyone.

Private sector organisations were more likely to offer financial or other incentives to those not taking any sickness absence; for example, one organisation entered employees with 100 per cent attendance records into a prize draw to win $£ 500$ while another offered employees the chance to win a car.

\section{The model of presenteeism}

The model of presenteeism derived from the previous literature (Figure 1) indicates a number of factors underlying an individual's decision to either attend or be absent from work when feeling unwell. Previous research has tended to focus on either workplace-related factors or the internal pressures individuals' experience when deliberating over whether to take time off for being sick. Often, these factors have been examined in isolation from one another, without an examination of how they might converge to produce different individual choices, in different situations. In testing the model, we aim to apply the findings of this study, which examined a wide range of causes of presenteeism in different workplaces, including those related to the Institution and those that emanate from the Individual, in the development of an understanding of how complex and multi-dimensional presenteeism is.

\section{Institutionally mediated presenteeism (organisational pressures)}

Institutionally mediated presenteeism was related to the organisational context or the working environment (including policies, procedures, management style and approach, and workplace culture). 
Sickness absence and trigger policies Many interviewees perceived that their organisation's sickness absence policies and procedures compelled attendance at work, especially where sick pay was withdrawn, or there was a threat of disciplinary action or dismissal. This was very common at CS1, where this pressure was felt to contribute to low morale, lack of commitment to work, stress and anxiety. The most unpopular policy was the suspension of sick pay following a number of episodes of absence as it had the most wide-reaching implications, in and out of work, and was at the discretion of managers who were often perceived as being inconsistent in their approach.

I'm twenty and I've bought a house with my partner and obviously, being that you've got to pay your bills it does get quite stressful because you think to yourself as much as you might not feel well enough to go into work you've got to because you won't get paid and it's fifty pounds, a lot of money to lose for a day. (Employee, private sector; CS1)

Policies with 'trigger points' existed in all the case studies, but they appeared to create a great deal of pressure at CS1 where individuals approaching their third or fourth period of absence faced possible disciplinary procedures:

I do sympathise with people that are up to their second or third occasion of illness and they've had an interview with me and they've got absolutely desperate stomach ache, toothache whatever, they come into work and go home. (Manager, private sector: CS1)

Another manager concurred suggesting that because sick pay is at the manager's discretion, employees drag themselves to work when they are sick, adding that germs then may get spread to others. The discomfort experienced by some managers was evident, especially where there was an expectation that policies should be strictly enforced as in both CS1 and CS2. Employees often felt they were in a 'lose-lose' situation; if they were absent they would be penalised, but if they attended work they would be unable to perform their duties effectively and would suffer too in terms of their health and general well-being. This was also in evidence at other case studies. At CS6, for example, interviewees reported that sick colleagues felt 'bullied' or 'pressured' back to work by organisational procedures which involved a representative from the organisation coming to their home if they were absent.

Absence management by HR Much of the interview data suggest that there is a perception that HR are primarily focused on getting people into the workplace and keeping them there, even when employees and managers felt that a period of absence was a better course of action. At CS2, for example, interviewees perceived a lack of flexibility and understanding on the part of HR when it came to understanding job roles; while some employees can return to work earlier in less physically demanding roles, this may not be appropriate for others. Managers felt pressure from HR departments and senior management to 'effectively manage' sickness absence, and reduce 'unofficial' absences. Many felt uncomfortable with that role as they did not feel supported by senior managers and they lacked an understanding of why they were required to carry out certain tasks, such as return-to-work interviews. Although it appeared from the interviews and discussions that HR departments were keen to devolve more responsibility for absence management to managers, in many cases there was little or inadequate training in 'people management' and a great deal of inconsistency in managers' approach to the role. This was demonstrated by the comments of a manager who described one 
type of management approach, explaining how policies might reduce absence, while also appearing unaware of how coercive her comments might sound:

I think (if) the sickness and absence policies are being strictly worked and abided by then (staff will) be really worried about taking unofficial absence. I don't want people to come into work with germs and bugs and things. A genuine sickness, no problem. It's sporadic sickness, definitely, I want to stop because if you've got less people off sick, you're going to feel less pressurised and also not fear - shouldn't use the word fear - but they all know 'oh gosh I can't take this sporadic sickness because I'll have another interview, may be a first formal or second formal, I cannot risk losing my job or something' whereas in the past they got away with it. So hopefully they'll think there's more hard line management really. (Senior manager, public sector, CS8)

Employees often viewed HR departments as being for policy implementation and absence management.

I feel that there's more of a push for people just to get them back into work, rather than supporting and it comes from an HR level and management level where it's a case of getting people back in and then we'll manage it afterwards. And that's where they see this penal side, where people think I'm being punished now for being ill. (Employee, private sector, CS6)

Occupational health $(\mathrm{OH})$ departments, where they were available, were more often viewed as being for the benefit of the employee and in particular to look after their health. Where $\mathrm{OH}$ departments worked well, employees sought out help, advice, health checks and felt 'looked after'. In contrast, those with a less high profile or proactive role were viewed as being less supportive or the place that employees were sent if they had too many absences, or in the words of one employee 'the big stick'.

'Return-to-work' interviews Return-to-work interviews were often perceived as organisational 'tools' designed to get a person back to the workplace rather than a show of concern about the employee's well-being:

Colleagues that have come in with broken legs that should really be covered by health and safety but are being told 'Oh, that's OK you can stay' and then later being told that no way should they be in the workplace. I know from my own experience ... they lay quite heavily on you that if your sickness doesn't improve that they'll terminate, that your contract will be terminated. (Employee, public sector; CS2)

Employees who had experienced return-to-work interviews often reported that it felt like a 'telling off' rather than a means of receiving support. This often left employees feeling quite stressed on their return to work as they knew they would receive such an interview.

Management style and management of absence Just as employees disliked the way return-to-work interviews were conducted, some managers disliked their formality and the rigidity of policies:

I don't like our attendance policy, I don't like the pressure it puts on individuals to come into work when they're not feeling particularly well. I also don't like these 
back to work interviews because I think more of an informal chat is better, again it puts pressure on people to come back before they're ready maybe... and then they go off sick again and I believe we don't believe them when they say they're sick. (Line manager, public sector; CS2)

As this line manager suggests, the rigidity of procedures may not allow for a sensitive, supportive approach, and may support a general culture of not believing that employees are ill. Another senior manager at CS2 suggested that despite a reduction of 1 per cent in absence rates 'on paper' brought about by rigidly adhering to policies, there is evidence of some underlying problems with employees attending work sick, suffering stress and anxiety over their absence, not wanting to hit the trigger point and subsequently becoming more unwell.

How to manage absence was a major concern for managers. They demonstrated very different perceptions and views about this whole area, reflecting the issue of consistency of approach that emerged in all the case studies.

Probably like a lot of organisations, there are some people who have sickness who perhaps aren't entitled to it and it isn't challenged and that creates a culture where people feel the ability to ask for time off as a right.... The other side of the coin is, unfortunately, there are occasions where because of inept management some people don't get the support they need, and that to me is just poor management. (Senior manager, public sector; CS3)

Expressions in the above quote such as 'aren't entitled to it' and 'time off as a right' are perhaps indicative of a culture of institutional presenteeism, where managers view sickness absence as discretionary rather than an employees' 'right'. In another extract illustrating the different management approaches to absence and sickness, a manager tries to downplay employees' feelings of being ill, suggesting it was her role to encourage her staff to think positively:

Somebody will say 'oh, I've got flu', and I say 'oh, you've only got a cold, just take an aspirin or something' and try and buck them up a bit really yes.... you've got to try and egg people on and say 'oh no, you're not as bad as you seem'. (Manager, private sector; CS1)

This perception contrasted with those of other interviewees in the organisation, reflecting the diverse perspectives that underpin management style.

Bonuses, incentives and promotion prospects Bonuses and incentives were common at private sector companies for employees who did not take time off sick, or who met sales or other organisational targets. At the public sector sites, there were more concerns about promotion prospects and performance-related pay.

At CS1, for example, sales targets brought bonuses that enabled employees to increase their wages. Employees often relied on these to provide what they considered a reasonable living wage. If they were off sick, they were less likely to meet sales targets and, in addition, many were unlikely to receive sick pay.

I think not being paid for time off, that is obviously an incentive to come in - you can have time off, great, but you're not going to be paid for it. Again, not in our department but in (another) the fact that you're not coming in, you won't be making your policies, you won't be adding towards your bonus.... I think people do feel pressured to come back in just because of losing them. (Employee, CS1) 
Interviewees had mixed opinions regarding the offering of bonuses and incentives to discourage sickness absence. Some felt that staff that had 100 per cent attendance records should be rewarded by the organisation. However, many felt that the pursuit of incentives could make employees feel unduly pressured to attend work.

\begin{abstract}
there's those who just don't go sick, unless they're really ill and there are those who do go sick. So, how do you differentiate between those? I just don't know. Looking at numbers, if you've not been sick for a year, that's good really, isn't it, because obviously, you have felt poorly at times? So perhaps acknowledging that in some way, recognising that you've not been off work all year, appreciating that with letters.... or, you could do a draw for a holiday in Majorca or something like that. (Line manager, private sector, CS3)
\end{abstract}

Although this manager's comments suggest that he would like to reward employees' commitment, he fails to acknowledge that individuals vary in the extent to which they can tolerate being unwell without their performance being affected, and that people faced with the same symptoms might react differently.

At public sector organisations, a key concern was the maintenance of a good work profile to ensure long-term promotion prospects were not harmed.

In my experience, I think most people will struggle on, but there are a few people who won't make the effort. Most people will because they know if they're not there, somebody else has got to pick their work up and it just puts extra pressure on other people... I think HR monitor that as well. It's competency related pay.... part of that competency is the fact that your sickness level is below average (for the organisation) or below a certain level. So, if during the year your sickness level creeps above that, then you'll lose that entitlement. (Supervisor, public sector)

Workplace culture At CS3, older employees and managers taught younger ones that they should discuss problems, get them sorted out and turn up for work and that it was not the 'done thing' to go off sick. This was the 'work ethic' passed down from worker to worker and impacted on the decision to be absent

\title{
Personally mediated presenteeism (personal motivation)
}

Personally mediated presenteeism reflecting individuals' internal drivers (such as their moral perspective on letting their colleagues, clients and the organisation down; or how they perceived absence might impact on their own career prospects) was widespread. There was less evidence of personal commitment to the workplace at organisations where employees felt coerced into being at work, such as at CS1 where the impact of the policies and procedures played a much greater role.

Commitment to colleagues and clients Employees in the public sector case studies experienced greater conflicting emotions over whether to attend work when they felt ill than occurred in the private sector case studies. Many did not want to let down their colleagues by being absent. They felt strongly committed to their organisation and loyal to the team who they perceived they had left to cover their job or duties.

Commitment to work was also strong when the general public were involved, although employees often felt that attending work with what they saw as a contagious illness was at odds with their desire not to pass on germs. Employees ended up feeling guilty both for taking time off work and for attending when sick: 
some might argue, 'Oh, it's only a cold' but if you're going around somewhere where people are trying to get better is it a good thing? ... It's a job to decide at times. Sometimes if you feel a bit yucky and you think, 'I'm coughing and sneezing' or something like that, do you or don't you, come in? Well I don't like being off any more than what I have to be but you just feel a little bit of responsibility towards other people... you can pass it on to somebody on the Ward and they could pass it on to a patient. (Employee, public sector; CS2)

Numerous interviewees from all case studies expressed concern about employees attending work with short-term, contagious illnesses like colds, flu, stomach bugs, diarrhoea and sickness. It was frequently felt to be an irresponsible and undesirable practice that might result in more people becoming absent. Although the majority of employees and some managers categorically stated that if a person was ill they should not be at work, individuals' definitions over what constitutes a level of illness that prevents work varied.

Personal progress Some employees suggested that taking time off might be viewed as an indicator of poor performance and might impact on their promotion prospects.

'I don't want to blot my copy book'. (Employee, private sector, CS3)

The perception that taking time off sick as a sign of under-performance was not always explicitly stated, but was often subtly evident in informal conversations, particularly in private sector organisations. 'High pressure' environments, with little spare capacity to accommodate absence, where employees held very specific or specialised roles and needed to be present to carry out essential tasks for the organisation to function at full capacity, were more likely to have a workplace culture that supported presenteeism.

\section{Variants of presenteeism}

Our findings suggest that the 'model' of presenteeism (Figure 1) needs to be seen as dynamic set of interactions, fluctuating according to circumstances. Although an individual might at one time have a low threshold for presenteeism, displaying a laissez faire attitude to absence, at another time the threshold may be raised because of additional work pressures, or internal pressures. For example, at case study sites CS3 and CS4 employees were required to work large amounts of overtime to conduct maintenance and for which they earned extra money, attendance was expected and every employee had a designated role (so both individual and organisational motivators to work even though an employee might be unwell); at these times there was very little absence. Following such periods, absence often increased (reduced workplace pressure and less to lose personally if a day or two of sickness absence taken in order to recover from the period of hard work). It might be argued that presenteeism increased during the time of high pressure, when organisational expectations are much higher, whereas during less pressured times, presenteeism reduces and absenteeism may increase.

HR staff and managers also experienced conflicting pressures regarding presenteeism. Here, one HR officer weighs up the financial costs of absence against the human costs including the possible consequences of attending work unwell or unfit.

the cost of sickness absence... [includes] the hard costings in terms of monetary value but also the impact on other people. So if Jack's off sick then John who sits next to him will have to cover some of his work... That puts additional strain on John so as well as the financial costs, there's the human costs as well... (However) if somebody is signed off sick and is told not to work, medical advice, 
we do have people on site here, who will go against that advice and still come into work and insist that they're fit enough to do the job ... that's never an acceptable course of action.... if somebody makes an error of judgement which is caused in part by the fact that they're operating at less than 100 per cent of their normal efficiency and they make a serious error then the impact could be enormous, so we discourage that. (HR officer, private sector, CS4)

Institutionally mediated presenteeism was characteristic of work environments with strongly enforced organisational policies, as described in previous research (Aronsson et al., 2000; Grinyer and Singleton, 2000; Hansson et al., 2006). In such organisations, presenteeism is fuelled by the threat of withdrawal of sick pay, trigger policies which hint at disciplinary action, and return-to-work interviews that are seen as punitive rather than supportive. In addition, incentives, such as bonuses and risks to career prospects, provide a further source of potential stress.

Policies and procedures which make employees feel insecure about their financial stability, employment or promotion prospects may create stress, tension, low morale and, at worst, employee antipathy towards the organisation itself. This is likely to affect well-being in the workplace, not only bringing employees into work when unwell but also potentially reducing their overall productivity and adversely affecting the wider organisational climate.

Individuals returning to work on light or modified duties, or phased returns, following long-term absence, often did so because they had been encouraged by their employers to return or because their entitlement to sick pay was running out. Despite being back in the workplace and re-engaging with work, these individuals may not yet be fully 'productive'. Some interviewees were sceptical about the purpose of bringing such individuals back to work before they had fully recovered, believing it was to keep sickness absence figures low rather than supporting the rehabilitation of the employee.

If you're ill there's nothing you can do, you're ill. You can't come to work. But there's a lot of these come in on light duties. What I feel is, come in to keep the sickness level down. I've seen lads in here hobbling in and I said 'what are you doing in?' He just came in and did nothing. (Employee, private sector, CS4)

Although the colleague described in the above extract was said to have come to work and 'did nothing', it may be that his presence at work served a rehabilitative function, whereas sitting at home and 'doing nothing' could be socially isolating and delay the return to work. Such perceptions may also reflect the area in which the colleague worked; for example, it might be more feasible for employees with desk jobs to return to work sooner than those with manual or physical jobs. Examples where this was the case were seen in both CS1 and CS2, where, for example, a manager who had returned to work early following an accident felt he had returned too soon, but because most of his job was desk-based, he felt that he had not suffered any negative effects.

There was at times a lack of understanding on the part of employees and managers about the purpose and benefit of phased return and modified working. Those who had benefited from such arrangements perceived that they had been provided with good support and valued the flexibility and understanding on the part of the organisation and managers. Despite the employee working at less than full capacity, the organisation was felt to be supporting their recovery and enabling them to return to their social network in the workplace quicker. This might be seen as promoting a positive form of presenteeism. 
In contrast to the supported return to work approach discussed above, there were reports of employees feeling pressured to attend work despite not feeling they were ready or fully recovered. Such employees often felt unable to perform to their full capacity, and there appeared to be heightened feelings of stress, resentment and lack of organisational commitment. Some reported experiencing repeated problems with their health or had made themselves feel worse as they tried to struggle on.

Where work environments fostered personally mediated presenteeism there was a strong commitment to colleagues and clients and professional image. Personally mediated presenteeism was most common amongst public sector staff, although not exclusively.

The responsibilities associated with job role also influenced the decision to attend work, while others suggested taking absence was due to their commitment not to pass on illnesses to their colleagues or clients with whom they might work.

In contrast, there was much evidence at CS1 and CS2 that employees routinely attended work suffering from short-term ailments such as coughs, colds and stomach bugs. Many interviewees felt that they risked passing on illnesses to their colleagues and felt that their own performance was adversely affected. Indeed we found that presenteeism was a significant cause of poor performance and increased absence and that presenteeism itself was often seen as irresponsible by employees and line managers alike:

We don't expect them to come in if they're unwell, if they can spread any coughs, sneezes or any illness, diarrhoea, vomiting, we don't want them in the workplace till they're fit and healthy to work. (Assistant manager, CS2)

It was clear that a balance needed to be struck between the needs of the service and the needs of its workforce. An insensitive approach from managers, for example, was more likely to increase presenteeism and reduce employee motivation and well-being at work. With a workplace culture that consistently placed pressure on its employees to be in work, we might assume that employees would, despite being physically present, simply become mentally disengaged.

\section{DISCUSSION}

By studying nine diverse organisations, we were able to examine all of the concepts described in previous research and summarised in our model of presenteeism. Our findings supported the idea that there are two distinct types of presenteeism, institutionally mediated presenteesim, which was more prevalent in the private sector organisations and related to organisational pressures (Grinyer and Singleton, 2000; Chatterji and Tilley, 2002; Taylor et al., 2003; Hansson et al., 2006) and personally mediated presenteeism which was more common in the public sector organisations (Shamansky, 2002; Aronsson and Gustafsson, 2005; Dew et al., 2005; Dixon, 2005).

We also found that the model derived from the literature was too simplistic. We listened to a vast array of perspectives on presenteeism and it became evident that there are many mediating factors which will promote or discourage presenteeism in a number of different circumstances. These factors work on an individual level in a dynamic manner so that while one person in an organisation might be discouraged from practising presenteeism, another may not think twice about it.

Mediating factors, such as fear of passing on illness to colleagues and clients were common across all organisations, as was the perception that attending work when unfit was not acceptable as it may increase organisational illness and hinder recovery. In addition, we found that early rehabilitation of employees might have some positive aspects, the underlying 
reasoning (simply to reduce figures or to support employees at their own pace?) affected how positive the organisation was about such policies.

Other common factors were how supported an employee felt (by their manager and organisation as a whole), what the particular circumstances were in the organisation and what was required of a person at work (for example, would they be missed; would absence impact on others; would they lose money?)

Our study refines earlier research, elucidating the fluctuating nature of presenteeism, depending on individual and organisational context. Early rehabilitation policies, for example, support Black's (2008) assertion that work is good for us and for some, the policies did appear to benefit the organisation and employee alike, when implemented with mutual consent, support and understanding. Such policies may also increase employee commitment and feelings of well-being and could reduce the propensity for employees to become disengaged from the workplace. However, where the motivation is simply to reduce absence statistics they may increase the problem of presenteeism giving managers problems with running an effective and productive service.

The impact of presenteeism on productivity is, however, hard to measure. Although previous research has suggested that the loss of productivity is greater with presenteeism than absenteeism (Grinyer and Singleton, 2000; Dixon, 2005; Main et al., 2005; Caverley et al., 2007), this study demonstrates the complexity of the relationship between these two things. For example, in the case of rehabilitation, where policies are well thought-out and are designed to support the individual, the organisation accepts that the employee is going to be less productive for a while but this may contribute to investing in their long-term commitment. Both employee and organisation are entering a reciprocal arrangement, one by returning to work early, the other by providing support and helping re-socialise the employee; both reap the benefits in the long term (the organisation may be more efficient by doing this as it reduces staff turnover costs, retraining costs, promotes workforce stability, employee loyalty and group cohesiveness). Employees returning to work voluntarily and being supported back in to work have less opportunity to drop out of the work market or to lose their self-confidence, jobs or skills and social network that their workplace provides, while the organisation holds on to the established experience and expertise of that individual. Such linkages are hard to understand if absence and presence are treated simply as opposites.

In contrast, we also found a lack of overt employee support in several organisations. If an employee feels unwell but fears taking sickness absence, then this might increase the likelihood that they attend work but feel disengaged. This fits with Samuel and Wilson's (2007) finding that employers who encouraged staff to take sick leave and were sympathetic to their illness promoted higher staff morale and loyalty. Certainly in organisations with more proactive employee support and less pressure placed on absent employees, employee well-being and commitment to their work place appeared higher.

We would suggest that organisations tailor their solutions for different people, teams and settings within an organisation and use multiple ways of assessing productivity and efficiency, moving away from the tendency to use absence data, introducing measures which tap into employee attitudes and views of their own productivity and even ask how often they attend work sick.

This study has provided further evidence that there is complex decision-making that underlies sickness absence and presenteeism and has sought to dispel some of the negative connotations that the term presenteeism has gained in order to capture the subtle differences between voluntary and institutional presenteeism, those who are 'under-performing' in the 
workplace and the fact that some aspects of 'presenteeism' may be beneficial, a concept that has not been explored previously. It has offered an alternative future for presenteeism, one which recognises that, like absenteeism, some can be positive and some can be negative but that it is not the act itself that should be managed and controlled, but the organisation's response to those who are participating in it.

The organisations that agreed to participate in this study cannot be considered to be representative of those across the UK economy; for example, no small enterprises were included. They were, indeed, self-selected on the basis that they were keen to improve well-being at work and were at different stages of creating improved health and well-being packages in their workplace. The fact that presenteeism loomed large here suggests that it is more of a feature across the economy generally; there is some evidence that it may also be a feature of the recession that began in 2007. Future research would need to cover a range of other organisations to further assess the extent and determinants of presenteeism. The present article has identified some antecedents and has shown how presenteeism is deeply embedded in organisational practice.

\section{REFERENCES}

Aronsson, G. and Gustafsson, K. (2005). 'Sickness presenteeism: prevalence, attendance-pressure factors and an outline of a model for research'. Journal of Occupational and Environmental Medicine, 47: 9, 958-966.

Aronsson, G., Gustafsson, K. and Dallner, M. (2000). 'Sick but yet at work. An empirical study of sickness presenteeism'. Journal of Epidemiology and Community Health, 54: 502-509.

Black, C. (2008). Working for a Healthier Tomorrow, http:/ / www.workingforhealth.gov.uk/documents / working-for-a-healthier-tomorrow-tagged.pdf, accessed 18 November 2009.

Caverley, N., Barton Cunningham, J. and MacGregor, J.N. (2007). 'Sickness presenteeism, sickness absenteeism, and health following restructuring in a public service organization'. Journal of Management Studies, 44: 2, 304-319.

Chatterji, M. and Tilley, C.J. (2002). 'Sickness, absenteeism and sick pay'. Oxford Economic Papers, 54: 669-687.

CIPD Survey (2005). Absence Management, http://www.cipd.co.uk, accessed 18 November 2009.

Dew, K., Keefe, V. and Small, K. (2005). '“Choosing” to work when sick: workplace presenteeism'. Social Science and Medicine, 60: 2273-2282.

Dixon, K. (2005). 'Weighing the costs of presenteeism: recognise the signs and repair the damage of employee burnout'. Chief Executive-New York, 209: 22-23.

Grinyer, A. and Singleton, V. (2000). 'Sickness absence as risk-taking behaviour: a study of organisational and cultural factors in the public sector'. Health, Risk and Society, 2: 1, 7-21.

Hansson, M., Bostrom, C. and Harms-Ringdahl, K. (2006). 'Sickness absence and sickness attendance - what people with neck or back pain think'. Social Science and Medicine, 62: 2183-2195.

James, P., Cunningham, I. and Dibben, P. (2002). 'Absence management and the issues of job retention and return to work'. Human Resource Management Journal, 12: 2, 82-94.

Jenneh, T. (2006). 'Mondial UK reduces absence and increases productivity: a collaborative approach to managing health and well-being'. Human Resource Management International Digest, 14: 5, 31-33.

Kivimaki, M., Leino-Arjas, P., Luukkonen, R., Riihimaki, H., Vahtera, J. and Kirjonen, J. (2002). ‘Work stress and risk of cardiovascular mortality: prospective cohort study of industrial employees'. British Medical Journal, 326: 7369, 857.

Main, C., Glozier, N. and Wright, I. (2005). 'Validity of the HSE stress tool: an investigation within four organisations by the Corporate Health and Performance Group'. Occupational Medicine, 55: 3, 208-214.

Patton, M. (1990). Qualitative Evaluation and Research Methods, 2nd edn, Newbury Park, CA: Sage Publications. 
Pilette, P. (2005). 'Presenteeism in nursing: a clear and present danger to productivity'. Journal of Nursing Administration, 35: 6, 300-303.

Pollitt, D. (2006). 'Pressure management keeps down the stress at Toshiba UK: company strives to be among the UK's best employers'. Human Resource Management International Digest, 14: 5, 29-30.

Pollitt, D. (2007). 'Cadbury's runs smoothly under pressure: wellness programme keeps IT project on track'. Human Resource Management International Digest, 15: 1, 14-16.

Ramsey, R. (2006). "'Presenteeism" a new problem in the workplace'. Supervision, 67: 8, 14-17.

Samuel, R.J. and Wilson, L.M. (2007). 'Is presenteeism hurting your workforce?'. Employee Benefit Plan Review, 61: 11, 5-7.

Schultz, A. and Edington, D.W. (2007). 'Employee health and presenteeism: a systematic review'. Journal of Occupational Rehabilitation, 17: 3, 547-579.

Shamansky, S.L. (2002). 'Editorial: presenteeism ... or when being there is not being there'. Public Health Nursing-Cambridge, 19: 2, 79-80.

Steers, R. and Rhodes, S. (1978). 'Major influences on employee attendance: a process model'. Journal of Applied Psychology, 63: 391-407.

Taylor, P., Baldry, C., Bain, B. and Ellis, V. (2003). " "A unique working environment": health, sickness and absence management in UK call centres'. Work, Employment and Society, 17: 3, 435-458.

Unison (1999). Tackling Sick. Absence Policies, http://www.unison.org.uk, accessed 18 November 2009.

Van Amelsvoort, L.G.P.M., Spigt, M.G., Swaen, G.M.H. and Kant, I. (2006). 'Leisure time physical activity and sickness absenteeism; a prospective study'. Occupational Medicine, 56: 3, 210-212.

Waddell, G. and Burton, K. (2006). Is Work Good for Your Health and Wellbeing?, London: TSO. 